P81 (continued)

Study Design, Setting, Participants, Intervention: Process evaluation of a 6-month community-randomized intervention trial: Strong Hearts, Healthy Communities (SHHC). A total of 101 women from eight rural towns were enrolled in the SHHC intervention program. Eligible participants were 40 years or older, sedentary, and overweight or obese. The 48-class program included strength training, aerobic exercise, nutrition education, and civic engagement activities.

Outcome Measures and Analysis: Program leaders completed after-class surveys to assess fidelity, dose delivered, and attendance. Program satisfaction and participation benefits were assessed through post-intervention surveys, leader interviews, and participant focus groups. Quantitative survey data were summarized using SAS v.9.4 and Microsoft Excel. Qualitative data were examined thematically using NVivo 11.

Results: Most sites reported high levels of adherence to the SHHC curriculum (average fidelity: $82 \%$, average dose delivered: $84 \%$ ). On average, participants attended $67 \%$ of the 48 classes, but this varied by site from $53 \%$ to $81 \%$. The majority of participants were satisfied with their experience and reported several benefits. Positive aspects of the program included group camaraderie, social support, and awareness building. Insufficient lesson time was the most common struggle reported. Recommendations included extending class length, expanding exercise variety, and enhancing discussion.

Conclusions and Implications: SHHC was wellreceived and deemed an acceptable approach by rural women enrolled in this CVD prevention program. Findings from this evaluation will inform program development and implementation for at-risk, underserved rural women and guide modification strategies for future dissemination. Funding: National Heart, Lung and Blood Institute (R01 HL120702).

\section{P82 Process Evaluation of a SNAP-Ed School and Community Gardens Policy, Systems, and Environment Initiative in New York State}

Alisha Gaines, PhD, CHES, againes@cornell.edu, Cornell University, Division of Nutritional Sciences, 342A MVR Hall, Reservoir Avenue, Ithaca, NY 14853;

Zoe Wakoff, MPH; Tisa F. Hill, MPH;

Jamie S. Dollahite, PhD

Background (Background, Rationale, Prior Research, and/or Theory): A unique SNAP-Ed policy, systems, and environment (PSE) initiative in New York State is designed to increase availability and consumption of fruit and vegetables using a three-tiered system of support including materials, technical assistance, and garden and nutrition education for gardens in high needs schools and communities. While evidence suggests garden programs may improve nutrition knowledge and behavior, process evaluation is essential to understanding the context of program effectiveness and implications for replicability, given variability in regional growing seasons and in local resources.

Objective: The purpose of this process evaluation was to determine the extent to which the garden PSE was implemented as intended and to identify facilitators and barriers to implementation in all 22 participating garden sites during FY 2017.

Study Design, Setting, Participants, Intervention: The mixed methods design included adaptation and review of program activity logs, monthly staff discussions, site visits, and 15 interviews conducted with 41 garden managers and participants.

Outcome Measures and Analysis: Quantified input, activity, and output data were compared with the program logic model and site-specific goals to assess fidelity. Interviews were audio recorded and transcribed verbatim. Qualitative data, including transcripts and field notes, were analyzed for themes related to program satisfaction and implementation successes and challenges.

Results: Staff were able to implement the program as planned in most sites. Exceptions included added technical support for five additional sites and limited programming in participating sites facing either internal (e.g. staff turnover) or external challenges (e.g. land lease revocation). Stakeholders expressed high levels of satisfaction with the program resources and staff. A few stakeholders requested additional planning and garden skills trainings, aligning with initial FY 2018 program plans.

Conclusions and Implications: Process evaluation results indicated achievement of most 2017 program goals and supported a shift in programming with current gardens to specifically foster sustainability. Results will complement forthcoming outcome evaluation data to inform revision of program materials and processes for statewide SNAP-Ed best practice discussions.

Funding: USDA.

\section{P83 Promoting Healthy Diet, Physical Activity and Life-Skills in High School Soccer Players: Results From the WAVE Ripples for Change Childhood Obesity Prevention 2-Year Intervention}

Yu Meng, MS, mengy@oregonstate.edu, Oregon State University, School of Biological and Population Health Sciences, College of Public Health and Human Sciences, HFC 3rd Floor, Corvallis, OR 97330; Siew Sun Wong, PhD; Melinda M. Manore, PhD, RD, CSSD, FACSM; John Schuna, PhD; Adam J. Branscum, PhD; Tonya Johnson, MPH, Oregon State University, Extension Family and Community Health Program; Megan Patten-Lopez, PhD, Oregon State University

Background (Background, Rationale, Prior Research, and/or Theory): Many high school (HS) athletes still lack sport nutrition knowledge and life-skills to overcome the challenging food environment around sports, thus, putting them at risk of developing obesity when

Continued on page 547 


\section{P83 (continued)}

they leave sports. The WAVE Ripples for Change (WAVE) project is a 2-year obesity prevention study, aimed to improve HS soccer players' sports nutrition knowledge, promote healthy eating, physical activity (PA) and life skill building.

Objective: Evaluate the impact of the WAVE project on HS soccer players' fruits and vegetable (FV), saturated fat, and added sugar intake; and their PA in- vs. out-ofsoccer season.

Study Design, Setting, Participants, Intervention: Based on geographic location (non-randomized), participants were assigned to the intervention group (IG) or comparison groups (CG) by school teams. IG received sports nutrition lessons, life-skill workshops, newsletters, and virtual experiential learning, while CG received none.

Outcome Measures and Analysis: Participants $(\mathrm{n}=388)$ completed pre-intervention demographic questionnaire, self-reported Block Food Frequency Questionnaire, and measured PA using Fitbit-Zip; 52\% completed post-intervention assessments. ANOVA was used to examine pre-/post intervention changes, and ANCOVA models to examine the between-group changes in diet and PA.

Results: The IG significantly decreased in added sugar (12 g/d) and saturated fat (3 g/d) intakes over the 2 years; the decrease of added sugar intake was significantly higher $(10 \mathrm{~g} / \mathrm{d})$ than the CG. No change in pre/post FV intake or differences between-groups. During soccer season, mean steps per day for all participants was 9,937 ( $\mathrm{M}=10,734$; $\mathrm{F}=9$,353), matching the step threshold consistent with compliance to the 2008 PA Guidelines for Americans. As expected, PA was 1,800 steps per day lower out-of-soccer season vs. in-season.

Conclusions and Implications: Over a 2-year period, the WAVE project successfully decreased added sugar and saturated fat intakes, and maintained FV intake in HS soccer players. Team sports play a critical role in keeping HS youth active.

Funding: NIFA.

\section{P84 Results of a Community Initiative in Rural Georgia: Healthy Retail and Social Marketing}

Amy DeLisio, MPH, RD, amy.delisio@wellness.phi.org, Public Health Institute, 1825 Bell Street; Suite 102, Sacramento, CA 95825; Kyli Gallington, MPH;

Suzanne Glenn, MS, HealthMPowers; Heather Rice, BS

Objective: To showcase methods and results of implementing a community initiative that focused on healthy retail changes and a social marketing campaign in three low income rural communities in Georgia.

Target Audience: SNAP-Ed practitioners and partners, nutrition educators, program implementers and evaluators, retail industry representatives.

Theory, Prior Research, Rationale: In 2016 HealthMPowers and the Public Health Institute collaborated to implement a community initiative to increase access to fresh fruits and vegetables (FV) at local retail- ers, establish an engaging social marketing campaign to influence behavior changes among residents, and ultimately increase purchasing and consumption of fresh FV. Extensive formative research in summer 2016 informed the social marketing campaign and healthy retail strategies: focus groups, key informant interviews, Communities of Excellence in Nutrition, Physical Activity, and Obesity Prevention (CX3) store environmental scans, and baseline shopper intercept surveys.

Description: Three low resource counties were selected for the initiative: Baldwin (Milledgeville), Washington (Sandersville) and Chatham (West Savannah) based on organizational readiness and local partnerships, existing obesity prevention infrastructure and community involvement efforts, and high poverty areas with strong participation in the Supplemental Nutrition Assistance Program and other federal food assistance nutrition programs. Based on formative research, the initiative developed a social marketing campaign, secured billboards in the communities, and provided participating retail stores with point of sales marketing materials and cues for consumers to easily identify healthier foods and beverages when shopping.

Evaluation: Survey respondents who recalled at least one social marketing ad also reported thinking more frequently about healthy food choices when deciding what to feed their family $(P=.0293)$. Seventy-one percent of residents who recalled at least one social marketing ad also reported purchasing fresh fruits and vegetables at least one time per week. About half of respondents indicated that they were eating more fruits and vegetables since last July (50.4\% and $45.1 \%$, respectively), and more than half (60.1\%) reported drinking less soda. Survey respondents that recalled at least one social marketing ad had a significantly higher self-efficacy score for fruit and vegetable consumption compared to respondents that did not recall social marketing ads $(P=.0022)$. In addition, the total number of social marketing images recalled by respondents was positively correlated with fruit and vegetable consumption self-efficacy, such that respondents who recalled more social marketing images had a higher selfefficacy score $(P=.0122)$.

Conclusions and Implications: Retail environmental changes coupled with social marketing campaigns can influence shoppers to make healthier choices. This program's success demonstrates to businesses that residents want healthier choices in their neighborhoods, making the case for future policies that reduce unhealthy food options and incentivize healthy ones.

Funding: USDA.

\section{P85 Self-Efficacy of Low-Income 5th Grade Students who Participated in a Policy, Systems, and Environmental Intervention}

Sabrina Hafner, BS, shafner@my.uri.edu, University of Rhode Island, 41 Lower College Road, Kingston, RI 02881; Geoffrey Greene, PhD, RDN; Linda Sebelia, MS, RDN; Maya Vadiveloo, PhD, RD; Adam Moore, PhD

Continued on page $S 48$ 